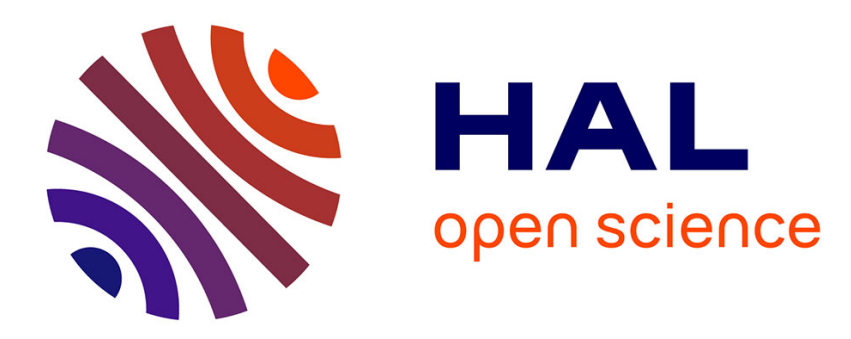

\title{
Ensemble d'enregistrement multiparamétrique à 28 digits binaires
}

\author{
R. Kamoun, R. Ballini
}

\section{To cite this version:}

R. Kamoun, R. Ballini. Ensemble d'enregistrement multiparamétrique à 28 digits binaires. Revue de Physique Appliquée, 1966, 1 (4), pp.293-297. 10.1051/rphysap:0196600104029300 jpa-00242750

\section{HAL Id: jpa-00242750 https://hal.science/jpa-00242750}

Submitted on 1 Jan 1966

HAL is a multi-disciplinary open access archive for the deposit and dissemination of scientific research documents, whether they are published or not. The documents may come from teaching and research institutions in France or abroad, or from public or private research centers.
L'archive ouverte pluridisciplinaire HAL, est destinée au dépôt et à la diffusion de documents scientifiques de niveau recherche, publiés ou non, émanant des établissements d'enseignement et de recherche français ou étrangers, des laboratoires publics ou privés. 


\title{
ENSEMBLE D'ENREGISTREMENT MULTIPARAMÉTRIQUE A 28 DIGITS BINAIRES
}

\author{
Par R. KAMOUN et R. BALLINI, \\ Centre d'Études Nucléaires, Saclay.
}

\begin{abstract}
Résumé. - Ce dispositif est réalisé par association d'élérnents standards. L'adjonction d'une mémoire tampon permet de l'utiliser pour un enregistrement de longue durée à faible taux de comptage.
\end{abstract}

Abstract. - This device is made of standard elements. A buffer memory allows it to be used it for long time recording at a low counting rate.

Des installations de même type, dont A. Pagès $[1,2,3]$, a donné les principes et réalisé les éléments essentiels avec ses collaborateurs, ont été utilisées dans diverses expériences ; l'une de ces expériences [4], sur l'étude des rayonnements de l'américium-241 a conduit à mettre au point l'ensemble décrit ci-après et sert d'exemple à l'appui de sa description.

Cet ensemble détecte, code, enregistre et traite l'information issue d'un événement nucléaire défini comme la détection, dans un même intervalle de temps de $200 \mathrm{~ns}$, d'une particule alpha et d'un rayonnement électromagnétique qui lui est associé par cette condition sur le temps.

On dispose de trois informations : énergie alpha, énergie gamma et temps qui s'est écoulé entre l'émission des deux rayonnements ; l'association de ces trois informations définit l'événement nucléaire.

Les circuits associés aux détecteurs alpha et gamma fournissent, pour chacun des détecteurs, une impulsion définissant l'instant de détection du rayonnement et une impulsion d'amplitude proportionnelle à l'énergie cédée au détecteur ; cette amplitude est traduite en un nombre entier par un convertisseur (ou traducteur) d'analogique en numérique.

L'intervalle de temps entre les deux impulsions définissant les instants de détection est aussi traduit par un nombre entier ; cette opération est actuellement effectuée sur l'impulsion délivrée par un convertisseur de temps en amplitude à partir des impulsions définissant les instants de détection. Les possibilités actuelles des traducteurs d'amplitude étant exploitées au maximum, chacun des spectres alpha et gamma est réparti sur 1024 canaux (10 digits binaires).

Notre enregistreur sur bande magnétique permettant soit 15 , soit $14 \times 2=28$, soit $13 \times 4=52$ digits, nous en avons utilisé 28 dont 8 réservés au spectre de temps.

Les nombres binaires traduisant les trois paramètres sont réunis dans 28 registres : leur association forme un nombre binaire unique qui représente l'événement à enregistrer.
L'enregistrement de l'événement occupe deux lignes sur une bande magnétique à 16 pistes dont une est réservée à un usage technique (pilote) et une autre, digit 15, sert à l'identification, par 0 ou 1 , de chacun des groupes de 14 digits.

La plus faible vitesse du dérouleur de bande magnétique sous sa forme actuelle est de $6 \mathrm{~mm} / \mathrm{s}$; comme il est possible d'inscrire 4 informations par millimètre (24 informations par seconde), la densité d'informations enregistrées est telle que la bande magnétique est mal utilisée au-dessous de quelques informations par seconde; or, dans certaines mesures en coïncidence, le nombre des événements intéressants est beaucoup plus faible encore.

D'autre part, à la vitesse de $6 \mathrm{~mm} / \mathrm{s}$, une bobine de $1500 \mathrm{~m}$ est déroulée en un peu moins de trois jours ; or, pour divérses expériences, comme celle qui a motivé cette installation, un enregistrement de durée beaucoup plus longue est nécessaire pour obtenir des résultats valables.

Nous avons donc procédé à un premier enregistrement dans une mémoire tampon ; l'occupation de la dernière adresse de la mémoire tampon déclenche le mouvement de la bande magnétique et le transfert du contenu de la mémoire tampon sur cette bande. Une première version $[5 a]$ a été réalisé avec un sélecteur à 100 canaux désaffecté. Une version, plus récente $[5 b]$ utilise un bloc-mémoire à 1024 ou 4096 canaux qui n'a subi aucune modification, ce qui permet de le réaffecter immédiatement à son usage habituel.

La lecture $\left(^{1}\right)$ de la bande magnétique consiste à identifier la première partie de l'information et à en mettre le contenu en mémoire jusqu'à l'arrivée de la deuxième partie; l'information initiale est alors reconstituée sous la forme d'un nombre binaire dont on se souvient qu'il est formé de trois nombres binaires juxtaposés.

Ainsi qu'il est exposé par ailleurs [1,2], l'infor-

(1) Pendant l'expérience une opération de lecture peut être effectuée sur l'information en cours d'enregistrement sans perturber cet enregistrement. 


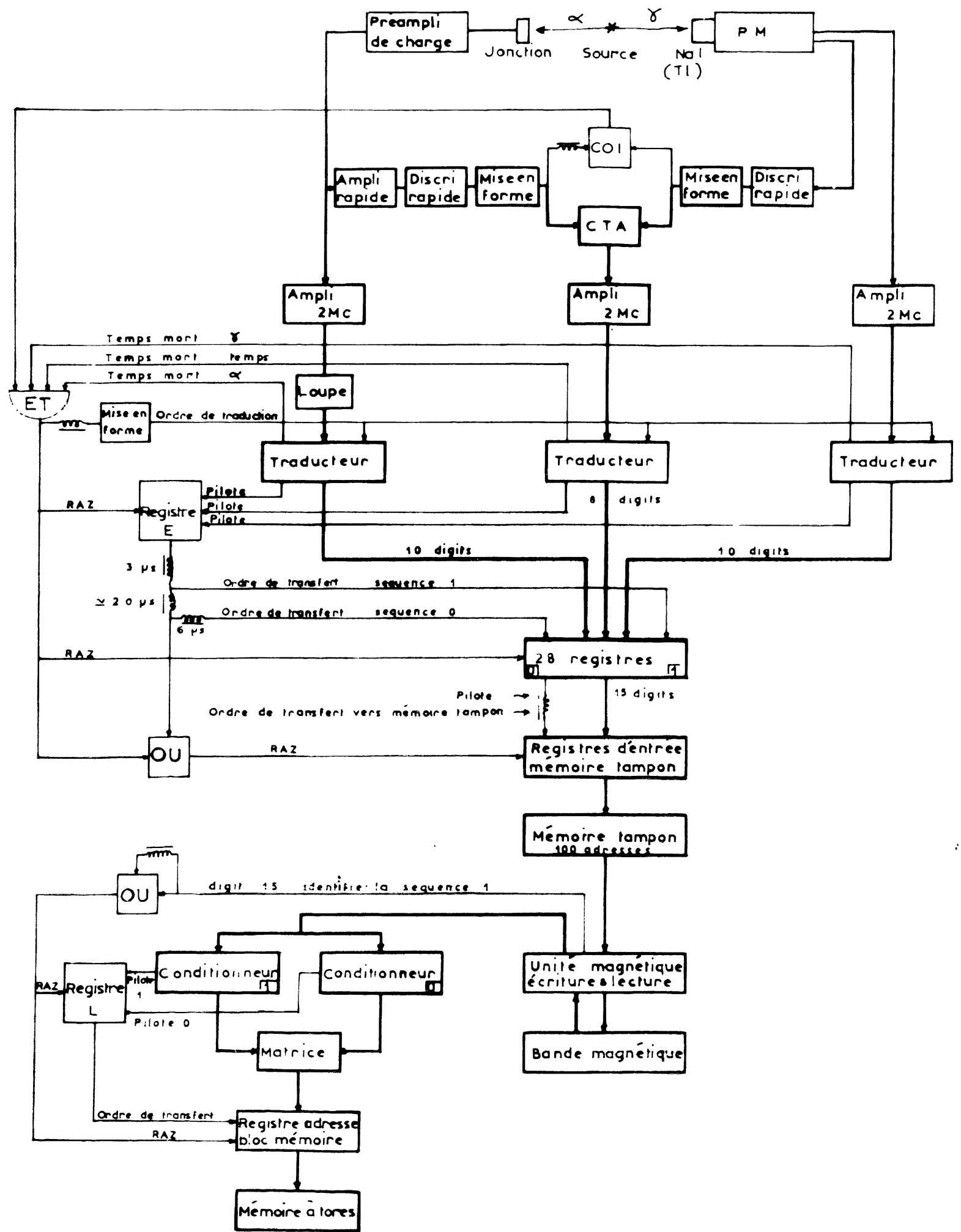

F:c. 1. - Ensemble d'analyse multiparamétrique à trois paramètres et 28 digits. 
mation n'est retenue que si elle respecte certaines conditions ; on augmente alors de 1 le contenu de l'adresse qui lui correspond, soit dans un bloc mémoire, soit dans une zone de mémoire d'un ordinateur si l'usage en est imposé par le traitement à faire subir aux informations. Un exemple de traitement par ordinateur (tracé de courbes de niveaux) est publié par ailleurs [6].

\section{Ordres de synchronisation des différentes opéra-} tions. - Les circuits utilisés sont constitués, pour l'essentiel, par des cartes à transistors de $10 \mathrm{~cm} \times 10 \mathrm{~cm}$ réalisées dans les laboratoires de A. Pagès et de J. Quidort. La première version du châssis de 28 registres a été étudiée par M. Avril puis reproduite par l'atelier de câblage du G. T. E. de Saclay avec introduction de divers circuits annexes dans le même châssis. L'utilisation de circuits élémentaires rend l'ensemble facilement adaptable à divers usages [7].

Ordres liés au début de traduction. - Les traducteurs ne doivent recevoir un ordre simultané de fonctionnement que si aucun d'entre eux n'est en train d'effectuer une opération antérieure. Sur la figure 1, représentant l'ensemble de l'installation, le rôle des divers organes apparaît ; chaque traducteur signale qu'il a reçu une information et qu'il est disponible pour l'analyser. Un circuit ET reçoit les signaux des traducteurs et, si nécessaire, le signal d'un circuit de coïncidence rapide. Le signal à la sortie du circuit ET est ainsi soumis au plus long des temps morts des traducteurs et il est utilisé :

- pour faire disparaître, par une remise à zéro des divers registres, tout ce qui subsiste de l'événement précédent ;

- pour donner l'ordre de traduction des paramètres à enregistrer.

Une autre possibilité ( fig. 2) consiste à soumettre à un temps? mort non cumulatif, supérieur au temps mort le plus long des ${ }_{-}^{-}$traducteurs, le signal délivré par le circuit $\mathrm{ET}$.

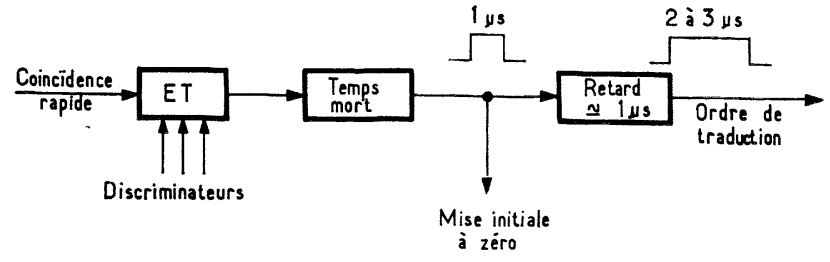

Fig. 2. - Un des procédés de production d'un ordre commun d'ouverture (le temps mort peut être produit par le circuit de la figure 3).

Ordres liés a la fin de traduction. - Comme on peut le voir sur la figure 1, chaque traducteur adresse en fin d'analyse le nombre binaire qu'il a déterminé à ceux des 28 registres auxquels il est connecté ; il adresse simultanément un avis (pilote) à un autre registre. Lorsque le dernier traducteur a adressé cet avis, il entraîne la production de deux signaux qui effectuent en deux temps le transfert hors des deux groupes de 14 registres, à travers des circuits OU sur une rangée commune de 14 digits. Un seul de ces signaux inscrit 1 sur le digit 15 en sortie.

Si la bande magnétique est précédée par la mémoire tampon déjà citée ou par un rythmeur (" derandomizer "), les opérations de transfert vers une de ces mémoires intermédiaires sont comprises dans le temps mort de l'ordre de traduction.

Sinon, le temps mis par la bande magnétique pour subir un déplacement suffisant (20 millisecondes pour une vitesse de $6 \mathrm{~mm} / \mathrm{s}$ ) doit séparer deux transferts successifs ; la figure 3 représente un montage qui a été utilisé pour imposer cet intervalle de

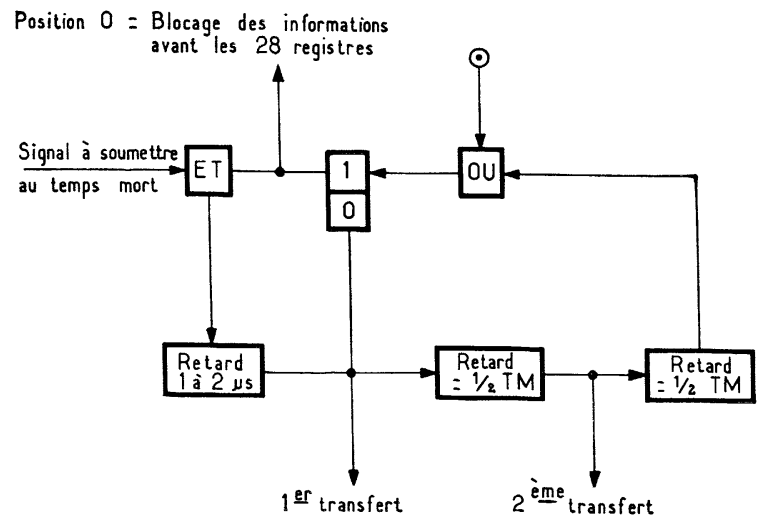

Fig. 3. - Temps mort non cumulatif. En raison du temps de restitution des monostables l'usage de deux retards en série s'impose même si on n'utilise pas le signal intermédiaire (deuxième transfert). Le circuit étant bloqué au cas, où, par exemple, le bistable se trouve à 0 lors de l'allumage, l'entrée inutilisée du circuit $O U$ permet le déverrouillage par un signal d'origine quelconque.

temps. Un autre procédé consisterait à imposer ce temps mort long dès l'ordre d'ouverture et à produire à partir de ce dernier, par deux retards successifs, les deux transferts. Nous avons préféré le procédé précédent car il permet de subordonner l'ordre de transfert à une opération de sélection effectuée après traduction en numérique : le temps mort long n'est alors déclenché que pour les informations sélectionnées. La figure 4 montre ainsi un montage utilisé pour diviser par 10 le nombre de certaines informations.

OrdRes liés a la SATURATION DE La MÉmoire taMPoN. - La chronologie des opérations déclanchées 
par la saturation de la mémoire tampon est représentée par la figure 5 .

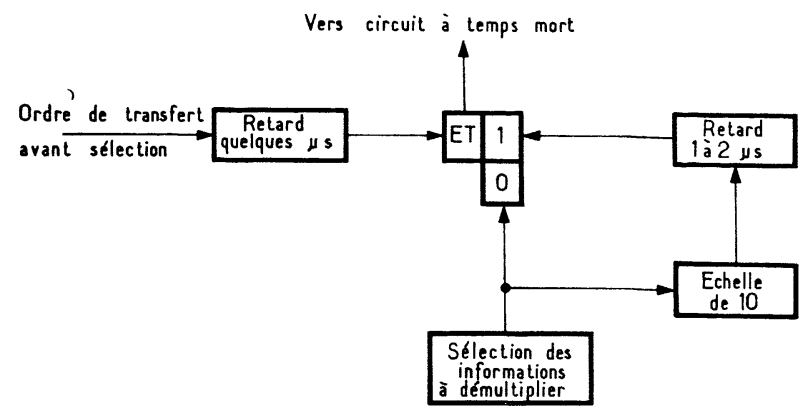

FIg. 4. -- Démultiplication par 10 de certaines informations.
Ordres liés a la lecture. - Le principe de l'opération est le suivant: la première partie d'une information constituée de 14 digits est identifiée soit par la présence du digit 15 (cas de la figure 1) soit par le pilote non accompagné du digit 15 (cas de la figure 6). Les registres intéressés par l'événement précédent sont alors remis à zéro; les 14 autres digits sont inscrits dans 14 registres si le digit 15 est absent, dans 14 autres s'il est présent. L'arrivée de la deuxième partie de l'information déclenche le transfert simultané hors des 28 registres; si une partie de l'information n'est pas arrivée en temps voulu, l'information incomplète est annulée par une mise générale à zéro des 28 registres, début de l'événement suivant. Dans une version réalisée en 1964 ( $f g$. 1) l'organe dési-

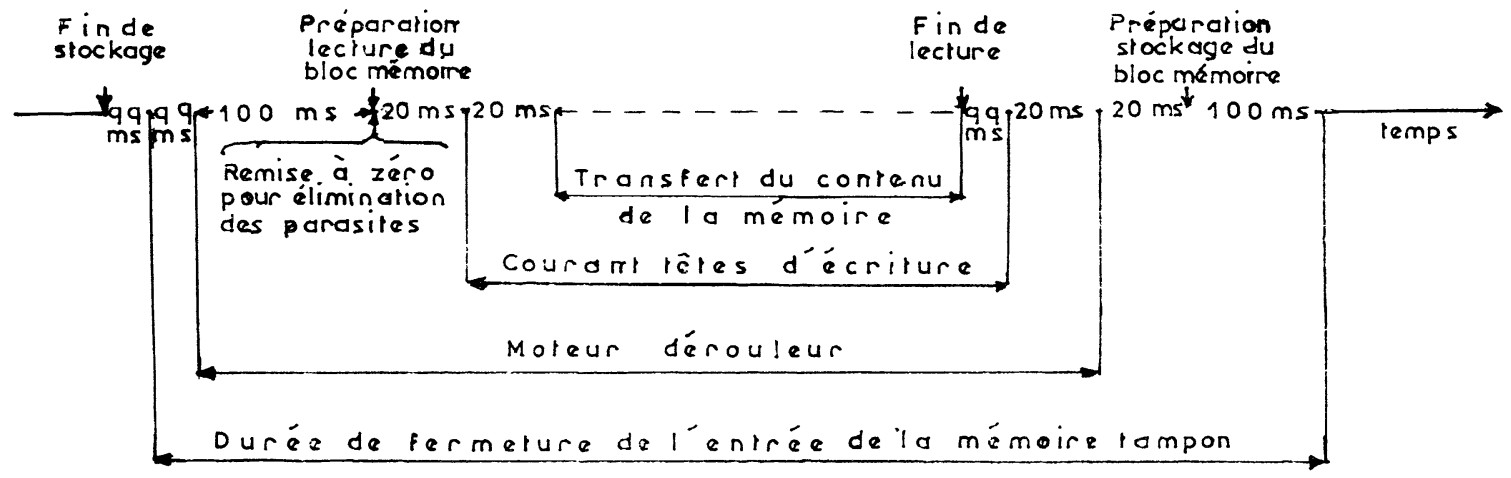

Fig. 5. - Transfert du contenu de la mémoire tampon.

Ce diagranıme est celui qui est prévu pour l'utilisation d'un bloc mémoire standard.

gné par «Sélection » dans la figure 6 est formé de deux conditionneurs à 15 digits connectés en parallèle à la sortie de l'unité magnétique ; la condition imposée au digit 15 est 0 pour l'un des conditionneurs, 1 pour l'autre. De plus l'ordre de transfert n'est produit que si les conditions imposées aux divers paramètres ( $\left.{ }^{2}\right)$ sont respectées.

Ce montage condensé a permis de remplacer pour la lecture le groupe de 28 registres de la figure 6 par les 12 éléments du registre d'adresse d'un bloc mémoire à 4096 canaux auxquels ne sont connectés, depuis la sortie des conditionneurs, que les digits concernés par les conditions imposées ; le transfert consiste alors à ajouter 1, à l'aide d'un signal extérieur, au contenu de l'adresse inscrite dans le registre d'adresses du bloc mémoire.

Il est à noter que l'usage d'un appareillage de ce

(2) Les deux conditionneurs ne travaillant pas au même instant dans ce dispositif provisoire, on ne pouvait pas imposer n'importe quelle condition à un paramètre réparti sur les deux groupes de 14 digits. genre n'est pas à opposer à celui d'un calculateur, mais qu'il peut le compléter en jouant le rôle d'organe périphérique capable d'autonomie.

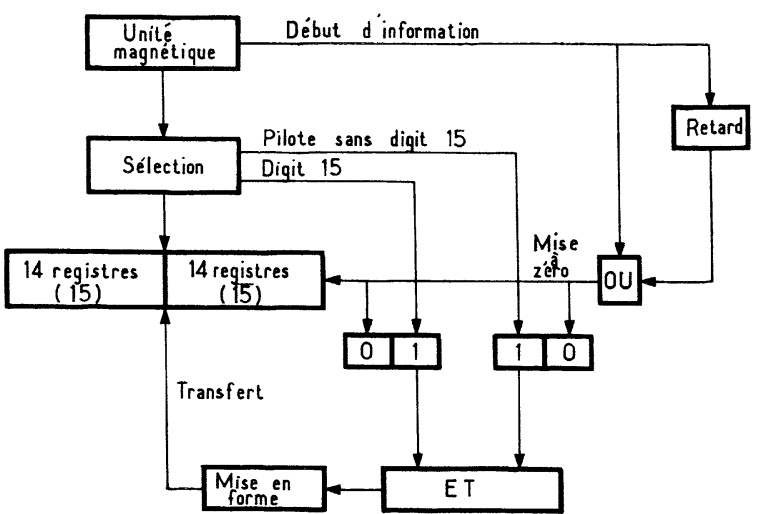

FIG. 6. - Schéma de principe de la lecture.

Manuscrit reçu le 20 juillet 1966. 


\section{RÉFÉRENCES}

11] Pages (A.), Coll. Intern. Electron. Nucl., Paris, 1958, p. 283.

[2] Pages (A.), Proc. Intern. Symp. Nucl. Electron., Belgrade, 1961, p. 289.

[3] Avril (M.) et Pages (A.), Proc. Conf. Karlsruhe, 1964 , p. 73.

[4] Kamoun (R.), Ballini (R.) et Duecknavorian (D.), Intern. Conf. Nucl. Phys., Paris, 1964, vol. 2, p. 571. [5a] Francois (B.), Rapport DEG/DIR/R-101, C. E. N., Saclay, 1965.

[5b] François (B.), à paraitre.

[6] Kamoun (R.), Faucon (A.) et Gluzman (P.), à paraître.

[7] Ballini (R.), J. Physique, 1964, 25, suppl. no 3, 31 A. 